\title{
Machine vision for various manipulation tasks
}

\section{Yukiyasu Domae}

Yukiyasu Domae, "Machine vision for various manipulation tasks," Proc. SPIE 10338, Thirteenth International Conference on Quality Control by Artificial Vision 2017, 1033815 (14 May 2017); doi: 10.1117/12.2277472

SPIE Event: The International Conference on Quality Control by Artificial Vision 2017, 2017, Tokyo, Japan 


\section{Plenary Talk III}

\section{Machine Vision for Various Manipulation Tasks}

\section{Dr. Yukiyasu Domae}

Advanced Technology R\&D Ctr., Mitsubishi Electric Corporation, Japan

\section{ABSTRACT}

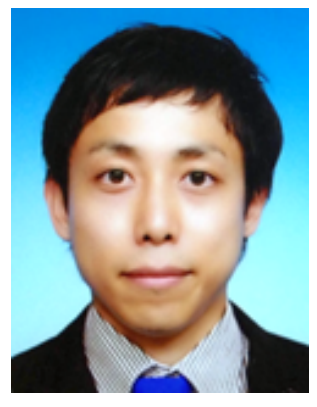

Bin-picking, re-grasping, pick-and-place, kitting, etc. There are many manipulation tasks in the fields of automation of factory, warehouse and so on. The main problem of the automation is that the target objects (items/parts) have various shapes, weights and surface materials. In my talk, I will show latest machine vision systems and algorithms against the problem.

\section{BIOGRAPHY}

Yukiyasu Domae is principal researcher of Mitsubishi Electric Corporation, and researcher of AIST, Japan. He is received the Ph.D. from the Hokkaido University in information science in 2012. He awarded R\&D100 award (2014), IPSJ Kiyasu special industrial achievement award (2014), best paper awards of JRM (2012) and RSJ (2016), Japanese robot award (2012) and Good Design Best 100 (2016) with the contribution of machine vision research and development. 